\title{
Perceptual grouping, input variability, and recall
}

\author{
CHARLES R. GRAH and HENRY C. ELLIS \\ University of New Mexico, Albuquerque, New Mexico 87131
}

\begin{abstract}
This experiment examined the role of internal group structure on recall of letter sequences under conditions of variable and constant stimulus input. Internal group structure is defined as the structure contained in each group of letters that results from the spatial grouping of letter sequences and is distinguished from overall stimulus structure, which refers to the structure contained in the intact letter sequence. While constant input led to better recall performance than did variable input when the internal group structure was high, the reverse was true when internal group structure was low. This was a different pattern of results than had been obtained previously with manipulations of external stimulus structure and suggested that internal group structure plays a qualitatively different role than does overall stimulus structure under conditions of variable stimulus input.
\end{abstract}

Several recent experiments have shown that stimulus variability facilitates recall. More specifically, these experiments have demonstrated that the presentation of variably grouped letter sequences leads to superior recall in comparison with sequences that are identically grouped on successive presentations (Ellis, Parente, Grah, \& Spiering, 1975; Ellis, Parente, \& Walker, 1974; Hunt, Parente, \& Ellis, 1974). These experiments have all used the same basic procedure of presenting subjects with letter sequences, such as B A NCOW, which can be spatially grouped in several ways, for example, BA NC OW. The spatial grouping of a given sequence of letters may be varied on successive presentations or, alternatively, may be held constant on each presentation, procedures which define varied vs constant input of information. As noted, the finding of interest is that, under a wide range of conditions, varied input produces substantially better recall of the sequences than does constant input.

An explanation of the "variability effect" in recall has been proposed by Ellis et al. (1974) in the form of a perceptual regrouping hypothesis. The principal assumption is that variation in the experimenter-imposed grouping of letter sequences over successive presentations leads a subject both to ignore eventually the experimenter-imposed structure and to discover the underlying structure (preexperimentally developed) and, hence, to encode this structure. Structure refers to the pronounceable, highly integrated, unspaced letter sequence. A necessary condition for the variability effect is that some minimal amount of underlying structure be present in the to-be-recalled sequence. A relationship between input structure and the variability effect has been nicely demonstrated by Parente

This research was supported by National Science Foundation Grant GB-27413X to the second author and by an NIMH training grant award to the first author. Reprint requests should be sent to Henry Ellis, Department of Psychology, University of New Mexico, Albuquerque, New Mexico 87131.
(1974). With highly structured materials, such as meaningful letter sequences that are easily pronounced, varied input led to superior recall. In contrast, with poorly structured material such as nonmeaningful letter sequences, varied input produced poorer recall.

Although it is clear that stimulus structure is an important factor in understanding the role of varied vs constant input on recall, it is important to make a logical distinction between two types of stimulus structure. One type of structure concerns the underlying structure present in an entire sequence of letters, which is the overall structure of the sequence. In contrast, a second type of structure is concerned with the particular groupings of letters within a sequence of letters. This latter type of structure is the internal structure or substructure of each intact grouping. This distinction is made concrete in the following example. Consider the grouped sequence of letters, CU PNE T. The overall structure of this sequence refers to the structure contained in the six letters when the grouping is ignored. In this case, it is obvious that the overall structure is quite high, since the six letters contain two words, CUP and NET. The internal group structure of the sequence refers to the structure of the three individual groupings of letters, CU, PNE, and T. Obviously the structure of these groupings is quite low.

The distinction between overall stimulus structure and internal group structure is an important one for the perceptual regrouping hypothesis. According to this hypothesis, a sequence with high overall structure is more likely to lead the subject to adopt the efficient encoding strategy of encoding the entire sequence. A prerequisite is that the subject must begin to ignore the experimenter-imposed groupings of the letters. It can be argued, however, that when the particular letter groupings are themselves high in structure (internal structure), subjects will tend to -encode the particular groupings as presented by the experimenter rather than look for some new overall grouping. If so, then this strategy would, under conditions of varied input, be 
likely to produce a number of varied and competing encodings which would tend to disrupt recall. Thus, it would appear that internal group structure and overall stimulus structure have qualitatively different effects on recall under the conditions of variable input. While the overall structure of a sequence determines the ease with which a subject may adopt the efficient strategy of encoding the entire sequence under varied input, the internal group structure may affect the ease with which the subject can ignore the experimenter-imposed organization of the letter sequence.

This analysis of internal group structure leads to a straightforward prediction that was tested in this experiment. When letter sequences which are sufficiently high in overall structure are broken into groupings that are themselves high in structure, then varied input by means of regrouping each letter sequence on subsequent presentations should disrupt recall performance relative to a condition in which the stimuli are input in a constant fashion. On the other hand, when the spatial grouping of letter sequences results in groups that are low in internal structure, then variable presentations of the letter sequence should support a higher level of recall than should constant presentations.

To test this prediciton, a number of letter sequences were constructed so that each could be spatially grouped in three different ways to produce three different strings of words (high internal group structure condition) and in three different ways to produce three different strings of nonword letter groupings (low internal group structure condition). The sequences were then presented grouped in the same way on each presentation (constant input condition) or were grouped differently on successive presentations (variable input condition).

\section{METHOD}

\section{Subjects and Design}

Eighty students from both high school and college volunteered for participation in this experiment. Twenty subjects were randomly assigned by order of appearance to one of four groups. Two types of stimulus input (constant and varied) and two levels of internal group structure (high and low) were manipulated as between-subject factors. All subjects were given eight study-test trials which, when combined with the two between-subject factors, yielded a 2 by 2 by 8 mixed-factor design.

\section{Procedure}

Four sequences of 18 letters each were constructed so that they could be spatially grouped into either sequences of words or nonwords (high and low internal group structure, respectively). Furthermore, there were three different ways in which each sequence could be grouped as either words or nonwords. All sequences, grouped in their different ways, are presented in Table 1.

All subjects were given eight study-test trials. During the study phase of each trial, subjects saw three presentations of the four word (or nonword) sequences. The order of presentation was block randomized in three blocks of four, with the restriction that each sequence occur in every block of four. The sequences were presented at the rate of one every $5 \mathrm{sec}$.
Table 1

Word and Nonword Sequences Which Describe High vs Low Internal Group Structure

High Structure Low Structure

CARPET CHILL YEARN OR

CARP ETCH ILL YEAR NOR

CAR PET CHILLY EARN OR

CA RPET CHI LLYEA RNOR CARPE TCHI LLYE ARNOR

OF TEN DAMP ASCENT ICE OFTEN DAM PASS ENTICE OFT END AMP ASS ENTICE

ASKING INCH ARM ANGLE ASK IN GIN CHAR MANGLE AS KING IN CHARM ANGLE

BATH ELAPSE AN EWE MIT BAT HE LAPSE ANEW EMIT BATHE LAP SEA NEW MIT CA RPE TCHIL LY EARNOR OFTF NDAMP ASCE NTI CE O FTEN DAMPA SSE NTICE OFTEND AMPA SSEN TICE

ASKIN GINC HARMA NGLE ASKI NGINC HAR MANG LE ASKI NGIN CHA RMAN GLE

B ATHE LAPSEA NE WEMIT BATHEL APS EAN EWEMIT BA THELA PSEA NE WEMIT

Subjects in the high internal group structure conditions always saw the sequences grouped as words, while those subjects in the low internal group structure conditions always saw the sequences grouped as nonwords. At no time did subjects see the unspaced letter sequence. In the varied input condition, the three different ways a sequence could be grouped according to the given internal group structure condition were presented on every trial. This meant that the spatial grouping of a sequence changed each time it was presented during a trial. In the constant input condition, the grouping of a sequence always remained the same, both within a trial and between trials. The particular grouping used for each sequence in the constant input conditions was determined by randomly selecting one of the alternative ways in which each sequence could be grouped.

During the testing phase of a trial, subjects were given a dittoed sheet with the first letter of each sequence. They were instructed to fill in all of the remaining letters in the appropriate order, guessing when they were not sure. The subjects were not required to group the sequences as they had been presented. No time limit was placed on the test, but subjects were encouraged to work as quickly as possible. The number of letters recalled in the correct position was recorded for each subject on every trial (total possible: 4 sequences $\times 18$ letters $=72$ ).

\section{RESULTS}

The data of primary interest are presented in Figure 1, which is a plot of the number of letters recalled in their correct position as a function of stimulus input condition, internal group structure condition, and trials. Two things are immediately apparent. First, when the letter sequences were spatially grouped as words (high internal group structure condition), recall was much greater than when the sequences were grouped as nonwords (low internal group structure condition) $[\mathrm{F}(1.76)=$ $16.473, \mathrm{p}<.001]$. Second, and most important, it is clear that input does not by itself have an effect $[F(1,76)<1.0]$ but does interact in a quite obvious fashion with internal group structure $[F(1,76)=10.739$, $\mathrm{p}<.005]$. While constant input produced a higher level of recall in the high internal group structure conditions, varied input produced a higher level of recall in the low internal group structure conditions. This pattern of results did not change as a function of practice, as evidenced by the nonsignificant three- 


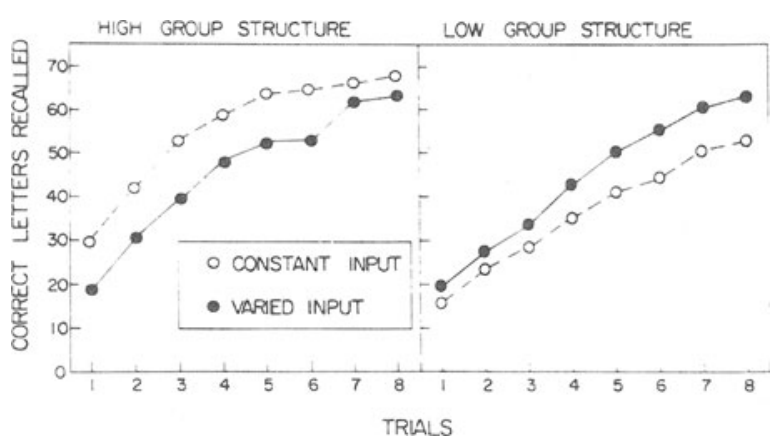

Figure 1. Number of letters recalled in their correct position as a function of stimulus input condition, internal group structure condition, and trials.

way interaction involving internal group structure, stimulus input, and trials $[\mathrm{F}(7,532)<1.0]$.

As expected, recall increased with practice $[F(7,532)=$ $232.21, \mathrm{p}<.001]$. The rate at which it increased, however, was not the same for all experimental conditions. For example, subjects who saw the letter sequences grouped as words learned at a faster rate than did subjects who saw the letter sequences grouped as nonwords $[F(7,532)=2.57, p<.05]$. Correct recall was also dependent upon the type of stimulus input (constant vs varied). While constant input produced higher levels of performance on the initial trials (1-6), varied input produced higher levels of performance on the latter trials $(7-8)[F(7,532)=2.22, \mathrm{p}<.05]$.

\section{DISCUSSION}

The major finding of this experiment was that varied input produced poorer recall performance than constant input when the structure of the groupings was high, but the reverse was true when the structure of the groupings was low. This pattern of results is distinctly different from that obtained by the manipulation of overall stimulus structure (Parente, 1974). While a high degree of overall structure enhances the "variability effect" in recall, a high degree of internal group structure produces the opposite results.

This pattern of results suggests that internal group structure plays a qualitatively different role than does overall structure. For example, internal group structure determines the extent to which subjects will attend to and encode the perceptually grouped sequence as presented by the experimenter. If the particular letter groupings contain a high degree of structure (are meaningful, easily pronounced, and easily integrated), the subject is most likely to encode the sequence as presented. When the grouping of a sequence changes on successive presentations and the resulting groupings remain high in structure, the subject is left with a number of variable and competing encodings of the same stimulus. On the other hand, when the structure of the groupings is low, the subject is not so bound to the organization imposed by the experimenter. Consequently, when the stimuli are variably presented, it is likely that the subject will ignore the groupings as presented and will attempt to reorganize the sequence in a more meaningful fashion. The extent to which the subject is successful in such an undertaking is dependent upon the amount of overall stimulus structure contained in the intact stimulus. In other words, while internal group structure determines the ease with which the subject can ignore the experimenter-imposed organization of a sequence, overall stimulus structure determines the ease with which the subject may reorganize the stimulus in a more meaningful and efficient way.

These data also account for the initially discrepant findings of Winzenz (1972, Experiment 5), in which variable presentations led to poorer recall performance than did constant presentations. In his experiment, Winzenz used stimuli similar to those used in the present experiment. The stimuli, however, were fractionated or grouped only as words and therefore constituted the high internal group structure conditions of the present experiment and, therefore, yielded the same pattern of results as did our high internal group structure conditions.

\section{REFERENCES}

Ellis, H. C., Parente, F. J., Grah, C. R., \& Spiering, K. Coding strategies, perceptual grouping, and the "variability effect" in free recall. Memory \& Cognition, 1975, 3, 226-232.

Ellis, H. C., Parente, F. J., \& Walker, C. W. Coding and varied input versus repetition in human memory. Journal of Experimental Psychology, 1974, 102, 284-290.

Hunt, R. R., Parente, F. J., \& Ellis, H. C. Transfer of coding strategies in free recall with constant and varied input. Journal of Experimental Psychology, 1974, 103, 619-624.

Parente, F. J. Stimulus structure and varied-constant input in recall of letter and digit sequences. Unpublished Master's thesis, University of New Mexico, 1974.

Winzenz, D. Group structures and coding in serial learning. Journal of Experimental Psychology, 1972, 92, 8-19.

(Received for publication January 12, 1977.) 